\title{
Surveillance, Methods and Evaluation
}

01 TAUPO BICYCLE STUDY: FOLLOW UP RESPONSE, PERSONAL CHARACTERISTICS AND INJURY OUTCOME

doi:10.1136/injuryprev-2012-040590a.1

S Tin Tin, A Woodward, S Ameratunga. School of Population Health, University of

Auckland, New Zealand

Background Web-based surveys are increasingly used as a resourceefficient approach to collecting research data. However, potential biases which may underlie response rates have received little attention in this context.

Aims This paper aimed to assess the associations between response to the first follow-up survey and baseline characteristics and injury outcomes in a cohort of cyclists.

Methods Baseline information was collected at recruitment in 2006 $(\mathrm{N}=2438)$ and $2008(\mathrm{~N}=190)$. A web-based follow-up survey was conducted in 2009/10. Injury outcome data were also collected through record linkage to insurance claims, hospital discharge and mortality data and police reports (covering the period from time of recruitment till 30 June 2011). Logistic regression models examined the association between baseline characteristics and response rates to the follow-up survey. Moreover, repeated event survival analysis was used to compare the risk of injury between responders and non-responders.

Results Of 2590 participants who resided in New Zealand, three died before the follow-up survey and 1526 completed the questionnaire $(59.0 \%)$. Older age group, female, higher level of education, greater number of years of cycling and cycling to work predicted higher response rates. Responders were more likely to have experienced bicycle crashes in general (adjusted hazard ratio (HR): 1.34; 95\% CI: 1.19-1.50), on road crashes (adjusted HR: 1.39; 95\% CI: 1.19-1.62) and collisions with a motor vehicle (adjusted HR: 1.33; 95\% CI: 1.15-1.54) during follow up.

Significance Differential follow-up response rates by demographic and cycling characteristics, as well as crash involvement require particular consideration in studies using web- based longitudinal data. 